\title{
A Riemannian Analysis of 3D Nose Shapes For Partial Human Biometrics
}

\author{
Hassen drira \\ LIFL (UMR USTL/CNRS 8022) \\ Université de Lille 1, France. \\ hassen.driraelifl.fr \\ Anuj Srivastava \\ Departement of Statistics \\ FSU, Tallahassee, FL 32306, USA. \\ http://stat.fsu.edu/ anuj/
}

\author{
Boulbaba Ben Amor \\ LIFL (UMR USTL/CNRS 8022) \\ Institut TELECOM ; TELECOM Lille 1, France. \\ http://www.telecom-lillel.eu/people/benamor/ \\ Mohamed Daoudi \\ LIFL (UMR USTL/CNRS 8022) \\ Institut TELECOM ; TELECOM Lille 1, France. \\ http://www.telecom-lillel.eu/people/daoudi/
}

\begin{abstract}
In this paper we explore the use of shapes of noses for performing partial human biometrics. The basic idea is to represent nasal surfaces using indexed collections of isocurves, and to analyze shapes of noses by comparing their corresponding curves. We extend past work in Riemannian analysis of shapes of closed curves in $\mathbb{R}^{3}$ to obtain a similar Riemannian analysis for nasal surfaces. In particular, we obtain algorithms for computing geodesics, computing statistical means, and stochastic clustering. We demonstrate these ideas in two application contexts : authentication and identification. We evaluate performances on a large database involving 2000 scans from FRGC v2 database, and present a hierarchical organization of nose databases to allow for efficient searches.
\end{abstract}

\section{Introduction}

Human biometrics has become an area of tremendous importance and potential. Although its growth in recent years have been motivated by security applications, one can safely expect an exponential growth in a general use of biometrics in our increasingly digital society. By human biometrics we mean the use of physiological characteristics, of human body parts and their appearances, to identify individual human beings in the course of their daily activities. The appearances of body parts, especially in imaged data, have a large variability and are influenced by their shapes, colors, illumination environment, presence of other parts, and so on. Therefore, the biometrics researchers have focused on body parts and images that try to minimize this variability within class (subjects) and maximize it across classes. Although several modalities, such as fingerprints, face images, iris, and gait, have been tried, none of them by itself may lead to a generic solution for a large population. Instead, an emerging consensus to develop a suite of different features (characteristics) and modalities, where each feature provides certain a partial information, such that their joint analysis may succeed for large populations. This has led to the notion of partial biometrics - the contribution of an individual feature/modality in identifying human beings.

The use of shapes of facial parts is an important example of this idea. Since 2D (visible light) images of faces are greatly susceptible to variations in the imaging environments (camera pose, illumination patterns, etc.), the researchers have argued for the need to use 3D face data, typically collected by laser scanners, for studying shapes of peoples' faces and using this data for biometrics. The output from laser scanners are minimally dependent on external environmental factors and provide faithful measurements of shapes of facial parts. Perhaps the only major remaining variability that is manifested within the same class, i.e. within the measurements of the same person, is the one introduced by changes in facial expressions. Facial expressions, such as smile, fear, and anger, etc, are prime indicators of the emotional state of a person. While they are important in estimating the mood of a person, for example in developing intelligent ambient systems, they have a lesser role in biometric applications. In fact, changes in facial expressions change the shapes of facial parts to some extent. We argue that this variability has become one of the most important issue in 3D face recognition as described in [2] and [9]. The other important challenge relates to data collection and imperfections introduced in that process. It is difficult to obtain pristine, continuous facial surfaces, or meshes representing such surfaces, with the current laser technology. One typically gets holes in the scanned data in locations of eyes, lips, and outside regions. These factors lead to a decrease 
in biometric performance. To handle these issues - shape variability due to facial expressions and presence of holes (missing data), we advocate the use of facial parts individually, especially for partial biometrics. The use of facial parts for biometrics is not new. For example, [16] and [3] have studied the use of human ear for recognition. The main advantage of using the ear, just like the nose [4], is that its shape does not change with facial expressions. Similarly, Faltemier et al. [5] have studied several different regions on a facial surface and have compared their contributions. We are not aware of any past work in an elastic (Riemannian) analysis of facial parts.

At the outset, the shape of the nose seems like a bad choice of feature for biometrics. The shapes of noses seem very similar to a human observer but we will support this choice using real data and automated techniques for shape analysis. We reiterate that this framework may not be sufficient for identifying human subjects across a vast population, but we argue for its role in shortlisting possible hypotheses so that a reduced hypothesis set can be evaluated using a more elaborate, multi-modal biometric system. The stability of nose data collection, the efficiency of nasal shape analysis, and the invariance of nasal shape to changes in facial expressions make it an important partial biometric.

Our approach for analyzing shapes of nasal surfaces is Riemannian. That is, we define a differentiable manifold, with a suitable Riemannian metric, whose elements represent individual noses. Our choice of representation is based on similar ideas followed previously for full faces use a collection of iso-curves to represent a surface and to compare surfaces by comparing their corresponding curves $[1,14]$. Our goals here are several : (i) Firstly, we want to evaluate the choice of (shape of) nose as a partial metric on a reasonably large database (involving 2000 scans), (ii) Secondly, we want to use Riemannian geometry to organize the database containing noses of a large population into a hierarchical (tree) so that efficient searches can be performed. Towards this end, we will explore the use of geodesic distances and Karcher means for clustering and averaging nasal surfaces.

The rest of this paper is organized as follows. In Section 2 we summarize some past work on comparing shapes of closed curves in $\mathbb{R}^{3}$ using an elastic metric, and apply this idea to analyze shapes of nasal surfaces. Also, we apply the classical Karcher mean computation to compute average of nasal surfaces. In Section 3, we study the use of geodesic distances in two biometric scenarios - identification and authentification. These experiments are based on comparing each query shape to each gallery shape. In the Section 4, we seek to make the database search more efficient, $O(\log (n))$ rather than $n$, by deriving a hierarchical database of gallery shapes.

\section{Riemannian Framework}

Our approach is to analyze shapes of nasal surfaces using shapes of iso-curves. In other words, we divide each surface into an indexed collection of simple, closed curves in $\mathbb{R}^{3}$ and the geometry of a surface is then studied using the geometries of the associated curves. Since these curves, called nasal curves, have been defined as level curves of an intrinsic distance function on the surface, their geometries in turn are invariant to the rigid transformation (rotation and translation) of the original surface. At least theoretically, these curves jointly contain all the information about the surface and one can go back-and-forth between the surface and the curves without any ambiguity. In practice, however, some information is lost when one works with a finite subset of these curves rather than the full continum.

In recent years, there have been several papers for studying shapes of continuous curves, the earlier papers were mainly concerned with curves in $\mathbb{R}^{2}[11,12]$. In this paper, we will follow the theory laid out by Joshi et al. [7, 8] for elastic shape analysis of continuous, closed curves in $\mathbb{R}^{n}$.

\subsection{Nasal Curves}

Let $N$ be a nasal surface, output of our preprocessing step (removing spikes, filling holes, and cropping nasal region) illustrated in Figure 1. Although, in practice, $N$ is a triangulated mesh, we start the discussion by assuming that it is a continuous surface. Let $D: N \longrightarrow \mathbb{R}^{+}$be a continuous geodesic map on $N$. Let $c_{\lambda}$ denote the level set of $D$, also called a nasal curve, for the value $\lambda \in D(N)$, i.e. $c_{\lambda}=\{p \in N \mid D(p)=G D(r, p)=\lambda\} \subset N$ where $r$ denotes the reference point (in our case the tip of the nose) and $G D(r, p)$ is the length of the shortest path from $\mathrm{r}$ to $\mathrm{p}$ on the mesh. We can reconstruct $N$ from these level curves according to $N=\cup_{\lambda} c_{\lambda}$ (see figure 1).

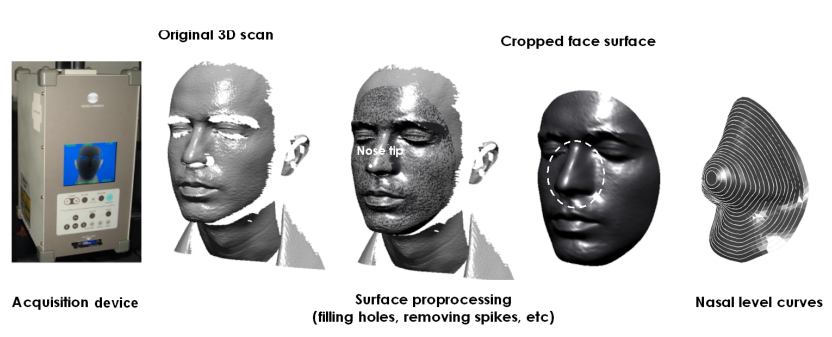

FIGURE 1. Automatic data preprocessing and nasal curves extraction

We start by considering a closed curve $\beta$ in $\mathbb{R}^{3}$. Since it is a closed curve, it is natural to parametrize it using $\beta: \mathbb{S}^{1} \rightarrow \mathbb{R}^{3}$. Note that the parametrization is not assumed to be arc-length; we allow a larger class of parametrizations for improved analysis. To analyze the shape of $\beta$, we shall represent it mathematically using a square-root 
velocity function (SRVF), denoted by $q(t)$, according to : $q(t) \doteq \frac{\dot{\beta}(t)}{\sqrt{\|\dot{\beta}(t)\|}}$. The classical elastic metric for comparing shapes of curves becomes the $\mathbb{L}^{2}$-metric under this representation [7]. This point is very important as it simplifies calculus of elastic metric to the well-known oneunder the $\mathbb{L}^{2}$-metric. In order to restrict our shape analysis to closed curves, we define the set :

$\mathcal{C}=\left\{q: \mathbb{S}^{1} \rightarrow \mathbb{R}^{3} \mid \int_{\mathbb{S}^{1}} q(t)\|q(t)\| d t=0\right\} \subset \mathbb{L}^{2}\left(\mathbb{S}^{1}, \mathbb{R}^{3}\right)$.

Here $\mathbb{L}^{2}\left(\mathbb{S}^{1}, \mathbb{R}^{3}\right)$ denotes the set of all functions from $\mathbb{S}^{1}$ to $\mathbb{R}^{3}$ that are square integrable. The quantity $\int_{\mathbb{S}^{1}} q(t)\|q(t)\| d t$ denotes the total displacement in $\mathbb{R}^{3}$ as one traverses along the curve from start to end. Setting it equal to zero is equivalent to having a closed curve. Therefore, $\mathcal{C}$ is the set of all closed curves in $\mathbb{R}^{3}$, each represented by its SRVF. Notice that the elements of $\mathcal{C}$ are allowed to have different lengths. Due to a non-linear (closure) constraint on its elements, $\mathcal{C}$ is a non-linear manifold. We can make it a Riemannian manifold by using the metric : for any $u, v \in T_{q}(\mathcal{C})$, we define :

$$
\langle u, v\rangle=\int_{\mathbb{S}^{1}}\langle u(t), v(t)\rangle d t .
$$

We have used the same notation for the Riemannian metric on $\mathcal{C}$ and the Euclidean metric in $\mathbb{R}^{3}$ hoping that the difference is made clear by the context.

So far we have described a set of closed curves and have endowed it with a Riemannian structure. Next we consider the issue of representing the shapes of these curves. It is easy to see that several elements of $\mathcal{C}$ can represent curves with the same shape. For example, if we rotate a curve in $\mathbb{R}^{3}$, we get a different SRVF but its shape remains unchanged. Another similar situation arises when a curve is reparametrized; a re-parameterization changes the SRVF of curve but not its shape. In order to handle this variability, we define orbits of the rotation group $S O(3)$ and the reparameterization group $\Gamma$ as the equivalence classes in $\mathcal{C}$. Here, $\Gamma$ is the set of all orientation-preserving diffeomorphisms of $\mathbb{S}^{1}$ (to itself) and the elements of $\Gamma$ are viewed as re-parameterization functions. For example, for a curve $\beta: \mathbb{S}^{1} \rightarrow \mathbb{R}^{3}$ and a function $\gamma: \mathbb{S}^{1} \rightarrow \mathbb{S}^{1}, \gamma \in \Gamma$, the curve $\beta(\gamma)$ is a re-parameterization of $\beta$. The corresponding SRVF changes according to $q(t) \mapsto \sqrt{\dot{\gamma}(t)} q(\gamma(t))$. We set the elements of the set :

$$
[q]=\{\sqrt{\dot{\gamma}(t)} O q(\gamma(t)) \mid O \in S O(3), \quad \gamma \in \Gamma\},
$$

to be equivalent from the perspective of shape analysis. The set of such equivalence classes, denoted by $\mathcal{S} \doteq$ $\mathcal{C} /(S O(3) \times \Gamma)$ is called the shape space of closed curves in $\mathbb{R}^{3}$. $\mathcal{S}$ inherits a Riemannian metric from the larger space $\mathcal{C}$ due to the quotient structure.

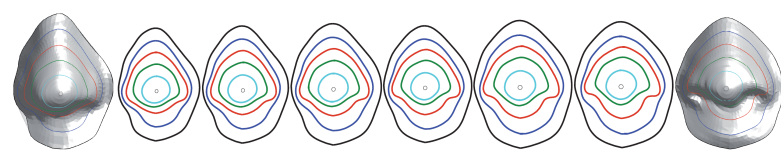

FIGURE 2. Examples of geodesics between curves

The main ingredient in comparing and analysing shapes of curves is the construction of a geodesic between any two elements of $\mathcal{S}$, under the Riemannian metric given in Eqn. 2. Given any two curves $\beta_{1}$ and $\beta_{2}$, represented by their SRVFs $q_{1}$ and $q_{2}$, we want to compute a geodesic path between the orbits $\left[q_{1}\right]$ and $\left[q_{2}\right]$ in the shape space $\mathcal{S}$. This task is accomplished using a path straightening approach which was introduced in [10]. The basic idea here is to connect the two points $\left[q_{1}\right]$ and $\left[q_{2}\right]$ by an arbitrary initial path $\alpha$ and to iteratively update this path using the negative gradient of an energy function $E[\alpha]=\frac{1}{2} \int_{s}\langle\dot{\alpha}(s), \dot{\alpha}(s)\rangle d s$. The interesting part is that the gradient of $E$ has been derived analytically and can be used directly for updating $\alpha$. As shown in [10], the critical points of $E$ are actually geodesic paths in $\mathcal{S}$. Thus, this gradient-based update leads to a critical point of $E$ which, in turn, is a geodesic path between the given points. Figure 2 shows two different surfaces of two different subjects and some examples of geodesic paths between level curves. The first and the last curves are the ones extracted from the two surfaces, and the intermediate curves denote equally-spaced points on the corresponding geodesic $\alpha$. These curves have been scaled to the same length to improve display of geodesics. We will use the notation $d\left(\left[q_{1}\right],\left[q_{2}\right]\right)$ to denote the geodesic distance, or the length of the geodesic in $\mathcal{S}$, between representations of the two curves $\beta_{1}$ and $\beta_{2}$.

\subsection{Nasal Surfaces}

Now we extend the framework from curves to surfaces. As mentioned earlier, we are going to represent a nose region surface $S$ with an indexed collection of the level curves of the $D$ function. That is, $N \leftrightarrow\left\{c_{\lambda}, \lambda \in\left[0, \lambda_{0}\right]\right\}$, where $c_{\lambda}$ is the level set associated with the distance $D$ equal to $\lambda$. Through this relation, each nasal surface has been represented as an element of the set $\mathcal{C}^{\left[0, \lambda_{0}\right]}$. In our framework, the shapes of any two noses are compared by comparing their corresponding nasal curves. Given any two nasal surfaces $N^{1}$ and $N^{2}$, and their associated curves $\left\{c_{\lambda}^{1}, \lambda \in\left[0, \lambda_{0}\right]\right\}$ and $\left\{c_{\lambda}^{2}, \lambda \in\left[0, \lambda_{0}\right]\right\}$, respectively, our idea is to compare the curves $c_{\lambda}^{1}$ and $c_{\lambda}^{2}$, and to accumulate these distances over all $\lambda$. For that, we define two possible metrics :

- Arithmetic mean : $d_{a}: \mathcal{C}^{\left[0, \lambda_{0}\right]} \times \mathcal{C}^{\left[0, \lambda_{0}\right]} \rightarrow \mathbb{R}_{\geq 0}$ given by $d_{a}\left(N^{1}, N^{2}\right)=\frac{1}{\lambda_{0}} \sum_{\lambda=1}^{\lambda_{0}} d\left(c_{\lambda}^{1}, c_{\lambda}^{2}\right)$.

- Geometric mean : $d_{g}: \mathcal{C}^{\left[0, \lambda_{0}\right]} \times \mathcal{C}^{\left[0, \lambda_{0}\right]} \rightarrow \mathbb{R}_{\geq 0}$, given by $d_{g}\left(N^{1}, N^{2}\right)=\left(\prod_{\lambda=1}^{L} d\left(c_{\lambda}^{1}, c_{\lambda}^{2}\right)\right)^{\left(1 / \lambda_{0}\right)}$. 
One advantage of a deformation-based comparisons of shapes of surfaces is that one has the actual deformations in addition to distances. (Also, the distances have important physical interpretations associated with them.) In particular, we have a geodesic path in $\mathcal{C}^{\left[0, \lambda_{0}\right]}$ between the two surfaces $N^{1}$ and $N^{2}$. This geodesic corresponds to the optimal elastic deformations of nasal curves and, thus, nasal surfaces from one to other. Shown in Figure 3 are examples of such geodesic paths - The first row involves nose regions of same subject but different scans, while the other rows show geodesics between nose regions that belong to different people.

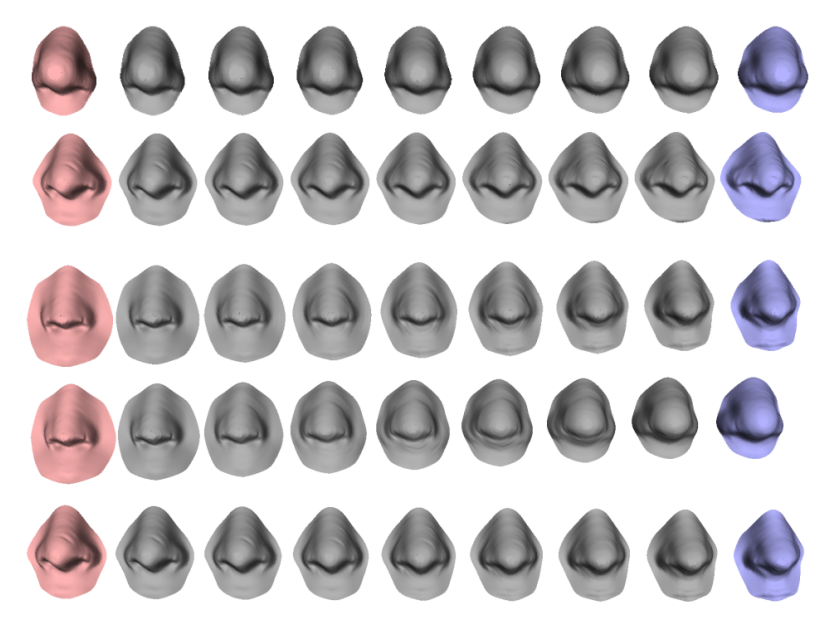

FIGURE 3. Geodesic paths between source and target noses. First two rows : intra-class paths, source and target with different expressions. Last three rows : inter-class paths.

\subsection{Mean Nasal Shape}

We can now to use our framework to calculate some statictics as the "mean" of surfaces. The Riemannian structure defined on the manifold of nose surfaces $\mathcal{C}^{\left[0, \lambda_{0}\right]}$ enables us to perform such statistical analysis for computing noses mean and variance. The Karcher mean utilizes the intrinsic geometry of the manifold to define and compute a mean on that manifold. It is defined as follows : Let $d_{s}\left(N^{i}, N^{j}\right)$ denote the length of the geodesic from $N^{i}$ to $N^{j}$ in $\mathcal{C}^{\left[0, \lambda_{0}\right]}$. In this particular case $d_{s}$ came from $d_{a}$. To calculate the Karcher mean of nose surfaces $\left\{N^{1}, \ldots, N^{n}\right\}$ in $\mathcal{C}^{\left[0, \lambda_{0}\right]}$, we define the variance function :

$$
\mathcal{V}: \mathcal{C}^{\left[0, \lambda_{0}\right]} \rightarrow \mathbb{R}, \mathcal{V}(N)=\sum_{i=1}^{n} d_{s}\left(N, N^{i}\right)^{2}
$$

The Karcher mean is then defined by :

$$
\bar{N}=\arg \min _{N \in \mathcal{C}^{\left[0, \lambda_{0}\right]}} \mathcal{V}(N)
$$

The intrinsic mean may not be unique, i.e. there may be a set of points in $\mathcal{C}^{\left[0, \lambda_{0}\right]}$ for which the minimizer of $\mathcal{V}$ is obtained. To interpret geometrically, $\bar{N}$ is an element of $\mathcal{C}^{\left[0, \lambda_{0}\right]}$, that has the smallest total deformation from all given nose surfaces.

We present a commonly used algorithm for finding Karcher mean for a given set of nose surfaces. This approach, presented in Algorithm 1, uses the gradient of $\mathcal{V}$ to iteratively update the current mean $\mu$.

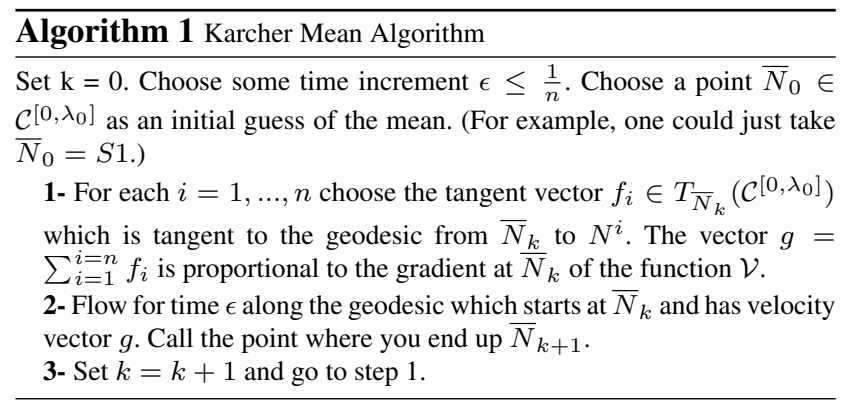

Since this is a gradient approach, it only ensures a local minimizer of the variance function $\mathcal{V}$. Several examples of using the Karcher mean to compute average faces are shown in Figures 4.

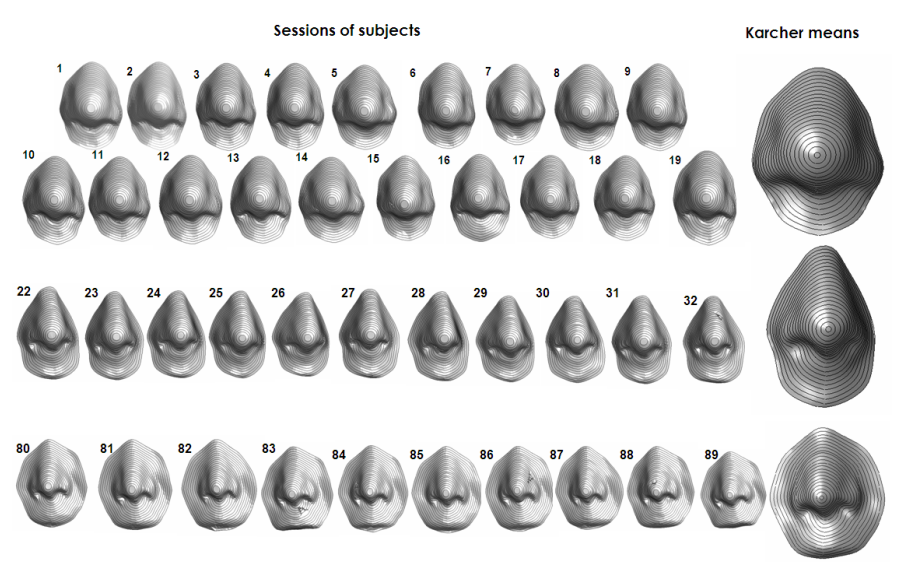

FIGURE 4. Examples of shapes and their karcher means.

What is the motivation for using statistical means of shapes in a biometric application? There are many possibilities. For example, one can develop a hierarchical organization of a population, where people (or observations) are first clustered into small groups and these clusters are replaced by their representatives, in this case Karcher means. Then, at the next higher level, one can cluster these means again and find their representative, and so on.

\section{Application to Human Biometrics}

In this section we present some experimental results in two different biometric scenario. These experiments use a 


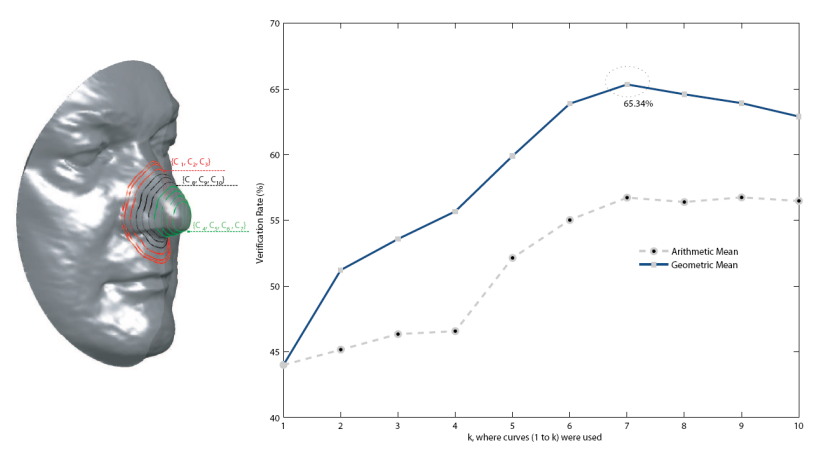

FIGURE 5. Verification Rate at $\mathrm{FAR}=0.1 \%$ using ( 1 to $\mathrm{k}$ ) curves

large subset, the first 2000 scans, of FRGC v2 dataset [13]. In order to produce results for both identification and authentication scenarios and to explore effect of the presence of facial expressions on performance, a distance matrix between a gallery and a query datasets is computed. The gallery contains 2000 sessions for 209 different subjects. The query set is identical to the gallery. The effectiveness of $d_{a}$ and $d_{g}$ in biometric applications generally increases with the number of curves used. But the question is how to choose the curves which can give best results? Indeed, in the nasal region, there are some areas like nasal cavities which exhibit undesired variability. Therefore, curves passing through these cavities could be eliminated from the experiment. This hypothesis will be tested and discussed in the following experiments. The results of identification and authentication scenarios will be presented separately.

Authentication Scenario : The first experiment involves authentication where the performance is measured using the verification rate (VR) for a given false acceptance rate (FAR) and with a receiver operating characteristic (ROC) curve that shows the trade-off between the verification and the false accept rates.

Figure 5 (right) shows a plot of VR at FAR $=0.1 \%$, computed using both the geometric and arithmetic means. The location of these curves on a nose are shown in the left. The best verification rate is obtained using the first seven curves and the geometric mean metric; it is approximately $65.34 \%$. As shown in the Figure 5, the verification rate increases with the number of used curves until $k=7$ curves are used and then it decreases. The curves which decrease the recognition rates pass through the nasal cavities. Moreover, we notice that the verification rates using the geometric mean $d_{g}$ are much better than those using the arithmetic mean $d_{a}$. So we choose this metric for further experiments. Figure 6 shows the ROC curves for experiments involving different number $k$ of curves. For $k<7$, the ROC curves using $1-k$ curves are higher for larger $k$. For $k>7$, this trend is reversed. So, using a larger number of curves improves the authentication performance until the seventh curve and, after that, results get worse.

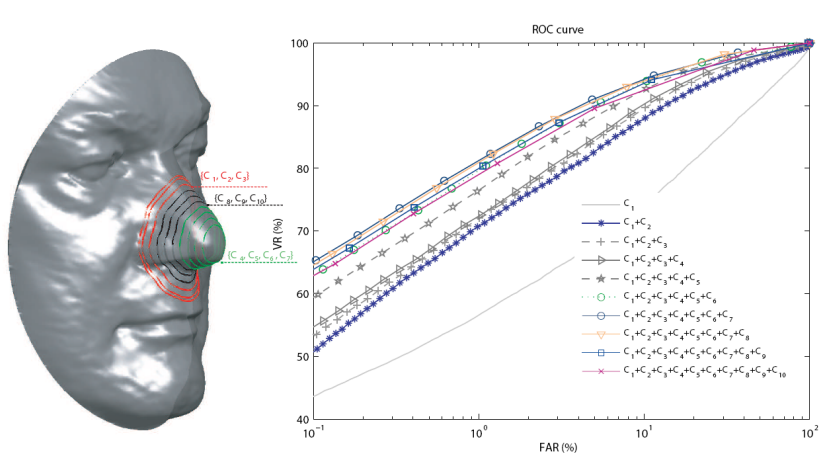

FIGURE 6. ROC curves using 1 to $\mathrm{k}$ curves

Identification Scenario : The second type of experiment is for identification for which the performance is quoted as a rank-one recognition rate. In this experiment the gallery set consists of neutral faces of 209 subjects. Figure 7 shows
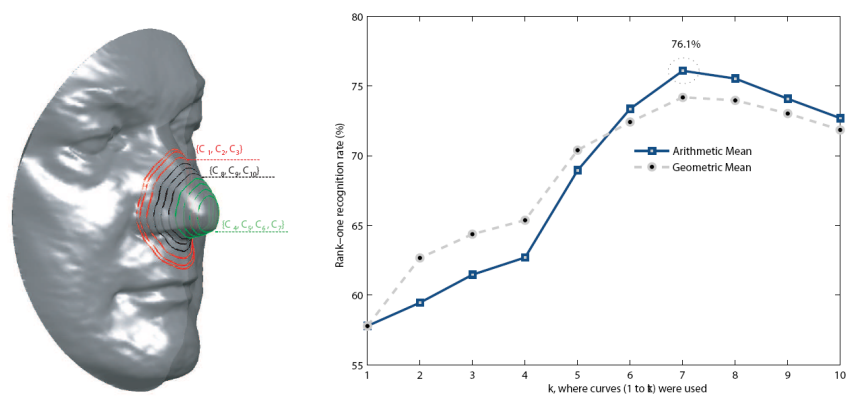

FIGURE 7. Rank one recognition rates using 1 to k curves

the rank-one recognition rates obtained using both geometric and arithmetic mean in the right and the locations of these curves on a nose in the left. We make the following remarks. First, we notice that the best recognition rate is obtained when using the arithmetic mean metric $d_{a}$. Actually, we obtain $76.1 \%$ using this metric using only seven curves. Second, we see that the recognition rate initially increases with the number of curves, reaches a peak and then starts decreasing, for both the metrics. The later curves which decrease the recognition rates are curves 8,9 and 10 and, as we see in the Figure 7 (left), these curves pass through the nasal cavities. Clearly, shapes of this region (made up of cavities) are not reliable and can be excluded from the analysis.

To summarize, our method allows us to compare the shapes of nose regions. By carefully selecting the set of stable curves, we are able to increase identification and verification rates. However, the identification scenario costs a 
lot in terms of time computation. In our case, to retrieve a shape in a dataset of 209 nasal surfaces, we need about 4 minutes. A hierarchical database organization can be performed in order to accelerate the identification process. We study an approach to cluster the gallery dataset using our framework.

\section{Hierarchical Gallery Organization}

One of the main goals for studying shapes of nose region is to conduct biometric searches where query is often compared to a set of gallery shapes. This comparison can be made more efficient if we can organize the gallery elements in form of a hierarchical database, i.e. a tree, where the comparisons are performed only at the nodes. To construct such a shape tree we need to be able to cluster similar shapes, and that is the problem we study next.

\subsection{Clustering Algorithm}

Consider the problem of clustering $n$ noses (in $\mathcal{C}^{\left[0, \lambda_{0}\right]}$ ) into $k$ clusters. A general approach is to form clusters in such a way that they minimize total "within-cluster" variance [15]. Let a configuration $C$ consists of clusters denoted by $C_{1}, C_{2}, \ldots, C_{k}$, and let $\mu_{i}$ s be the mean shapes in $C_{i} \mathrm{~s}$ and $n_{i} \mathrm{~s}$ be the sizes of $C_{i} \mathrm{~s}$. There are several cost functions used for clustering, e.g the sum of traces of covariances within clusters. However, the computation of means $\mu_{i} \mathrm{~s}$ of large shape clusters, and therefore their variances, is computationally expensive, especially when they are updated at every iteration. As a solution, one often uses a variation, called pairwise clustering [6], where the variance of a cluster is replaced by a scaled sum of distances (squared) between its elements :

$$
Q(C)=\sum_{i=1}^{k} \frac{2}{n_{i}}\left(\sum_{N^{a} \in C_{i}} \sum_{b<a, N^{b} \in C_{i}} d\left(N^{a}, N^{b}\right)^{2}\right) .
$$

We seek configurations that minimize $Q$, i.e., $C^{*}=$ $\operatorname{argmin} Q(C)$. Notice that the metric used is the arithmetic mean $d_{a}$. We will minimize the clustering cost using a Markov chain search process on the configuration space. The basic idea is to start with a configuration of $k$ clusters and reduce $Q$ by re-arranging shapes amongst the clusters. The re-arrangement is performed in a stochastic fashion using two kinds of moves. These moves are performed with probability proportional to the negative exponential of the $Q$ value of the resulting configuration. The two types of moves are following. (1) Move a shape : Here we select a shape randomly and re-assign it to another cluster. Let $Q_{j}^{(i)}$ be the clustering cost when a shape $N_{j}$ is re-assigned to the cluster $C_{i}$ keeping all other clusters fixed. If $N_{j}$ is not a singleton, i.e. not the only element in its cluster, then the transfer of $N_{j}$ to cluster $C_{i}$ is performed with probability :
$P_{M}(j, i ; T)=\frac{\exp \left(-Q_{j}^{(i)} / T\right)}{\sum_{i=1}^{k} \exp \left(-Q_{j}^{(i)} / T\right)} i=1,2, \ldots, k$. Here $T$ plays a role similar to temperature in simulated annealing. If $N_{j}$ is a singleton, then moving it is not allowed in order to fix the number of clusters at $k$. (2) Swap two shapes : Here we select two shapes randomly from two different clusters and swap them. Let $Q^{(1)}$ and $Q^{(2)}$ be the $Q$-values of the original configuration (before swapping) and the new configuration (after swapping), respectively. Then, swapping is performed with probability : $P_{S}(T)=\frac{\exp \left(-Q^{(2)} / T\right)}{\sum_{i=1}^{2} \exp \left(-Q^{(i)} / T\right)}$.

In order to seek global optimization, we have adopted a simulated annealing approach. Although simulated annealing and the random nature of the search help in avoiding local minima, the convergence to a global minimum is difficult to establish. The main steps of the algorithm is given by Algorithm 2.

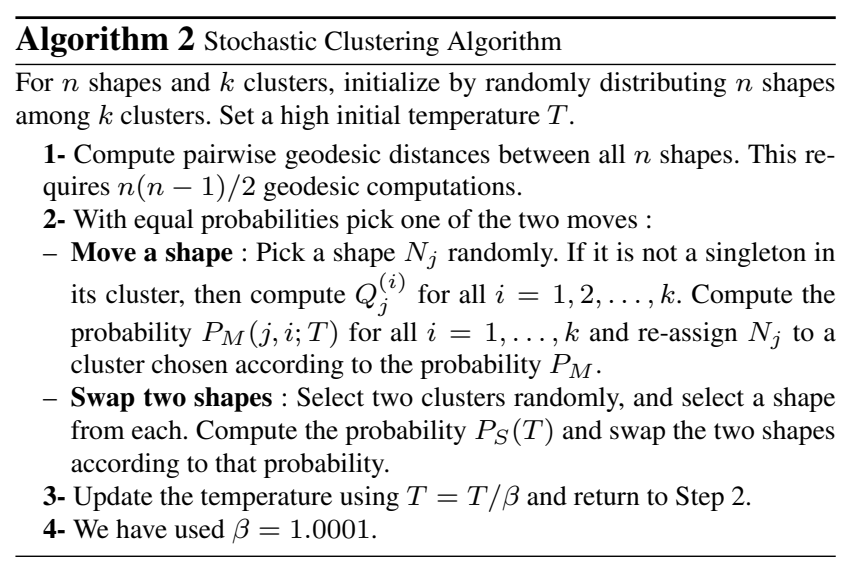

It is important to note that once the pairwise distances are computed, they are not computed again in the iterations. Secondly, unlike $k$-mean clustering, the mean shapes are never calculated in this clustering.

The algorithms for computing Karcher mean and clustering can be applied repeatedly for organizing a large database of human noses into a hierarchy that allows efficient searches during identification process. As an illustration of this idea, we consider 500 nose scans corresponding to 50 distinct subjects. These noses form the bottom layer of the hierarchy, called level E in Figure 8.Then, we compute Karcher mean shapes (representative shapes) for each person to obtain shapes at level D. These shapes are further clustered together and a Karcher mean is computed for each cluster. These mean shapes form the level $\mathrm{C}$ of the hierarchy. Repeating this idea a few times, we reach the top of the tree whith only one shape. We obtain so the final tree shown in Figure 8.If we follow a path from top to bottom of the tree, we see the shapes getting more particularized to groups and then to individuals as illustrated in Figure 8. 


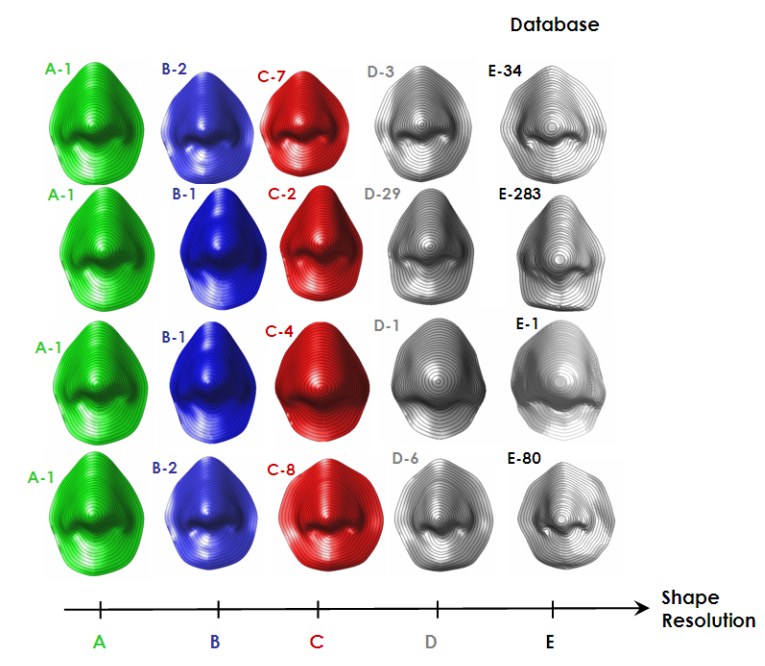

FIGURE 8. Paths from top to buttom in the tree show increasing shape resolutions

\subsection{Hierarchical Shape Retrieval}

Once the tree is formed, one can use this representation of data to conduct biometric search in order to reduce computation time, especially in identification scenario, which needs a comparison of the query shape to the whole gallery dataset. In view of this structure, a natural way is to start at the top, compare the query with the shapes at each level, and proceed down the branch that leads to the closest shape. At any level of the tree, there is a number, say $h$, of possible shapes and our goal is to find the shape that matches the query best. Figure 9 illustrates 2 examples of retrieval using the hirarchical organization of the dataset. For Q1 for example, the query nasal shape (at the top) is first compared to the shapes of level $B$. As it is closer to shape $B_{1}$, we proceed down the corresponding branch. There, the query proceeds down the branch of the shape $C_{2}$ as this shape is the closest one in this level to the query. The decision of the retrieval is given after comparison with shapes at level D. In this case the query is matched to the shape $D_{2}$. Actually, the shape $D_{2}$ is the mean shape of nasal shapes of the same person of the query. Notice that nasal shapes of each person at level $E$ are represented by their mean at level $D$. The last match decides in which cluster the query belongs. According to our tree, time computation for shape retrieval is approximately 3-10 times faster than exhaustive comparison as given by table 1 for 11 examples of queries. Results on a larger experimental set will be presented in the final versions.

\section{Conclusions}

In this paper, we have proposed a geometric analysis of 3D nasal shapes for the use in partial human biometrics.
TABLE 1. Average time computation and retrieval results

\begin{tabular}{|c|l|l|l|}
\hline Query & Exhaustive & Hierarchical & Result \\
\hline$Q_{i}, i \in[1 . .9]$ & $75 \mathrm{~s}$ & $16.05 \mathrm{~s}$ & correct \\
\hline$Q_{10}, Q_{11}$ & $75 \mathrm{~s}$ & $16.5 \mathrm{~s}$ & incorrect \\
\hline
\end{tabular}

The main tool presented in this paper is the construction of geodesic paths between arbitrary two nasal surfaces. The length of a geodesic between any two nasal surfaces is computed as the geodesic length between a set of their nasal curves. In particular, we have presented results for computing geodesics, computing statistical means and stochastic clustering to perform hierarchical clustering. We have demonstrated these ideas in two application contexts : the authentication and the identifiation biometric scenarios using nasal shapes on a large dataset involving 2000 scans, and hierarchical organization of noses gallery to allow efficient searches.

\section{Acknowledgements}

This research is supported in part by the ANR under the project ANR-07-SESU-004 and the Contrat de Projet EtatRégion (CPER) Région Nord-Pas de Calais Ambient Intelligence and partially supported by the following grants : ARO W911NF-04-01-0268 and AFOSR FA9550-06-1-0324 to Anuj Srivastava. Additionally, Anuj Srivastava was supported by visiting professorships from University of Lille I and CNRS in summers of 2007-2009.

\section{Références}

[1] B. B. Amor, H. Drira, L. Ballihi, A. Srivastava, and M. Daoudi. An experimental illustration of $3 \mathrm{~d}$ facial shape analysis under facial expressions. Annals of telecommunications, 64(5-6) :369-379, 2009.

[2] A. M. Bronstein, M. Bronstein, and R. Kimmel. Threedimensional face recognition. International Journal of Computer Vision, 64(1) :5-30, 2005.

[3] H. Chen and B. Bhanu. Human ear recognition in 3d. IEEE Transactions on Pattern Analysis and Machine Intelligence, 29(4) :718-737, 2007.

[4] H. Drira, B. B. Amor, M. Daoudi, and A. Srivastava. Nasal region contribution in $3 \mathrm{~d}$ face biometrics using shape analysis framework. In M. Tistarelli and M. S. Nixon, editors, $A d$ vances in Biometrics, Third International Conference, ICB 2009, Alghero, Italy, June 2-5, 2009. Proceedings, volume 5558 of Lecture Notes in Computer Science, pages 357-366. Springer, 2009.

[5] T. Faltemier, K. Bowyer, and P. Flynn. A region ensemble for 3-d face recognition. IEEE Transactions on Information Forensics and Security, 3(1):62-73, 2008.

[6] T. Hofmann and J. M. Buhmann. Pairwise data clustering by deterministic annealing. IEEE Transactions on Pattern Analysis and Machine Intelligence, 19(1) :1-14, 1997. 


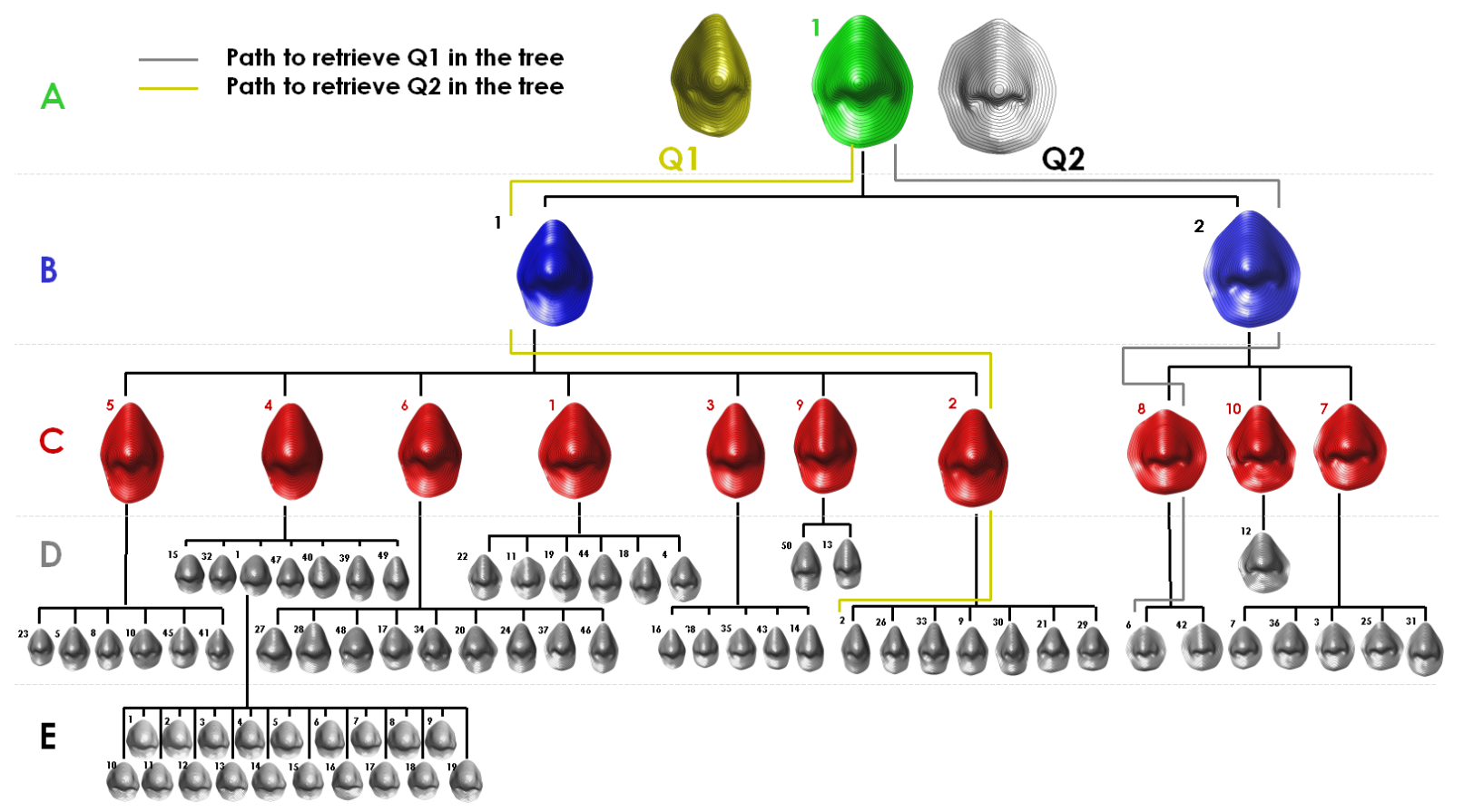

FIGURE 9. Examples of paths parsed by two queries across the tree.

[7] S. H. Joshi, E. Klassen, A. Srivastava, and I. H. Jermyn. A novel representation for efficient computation of geodesics between $n$-dimensional curves. In IEEE CVPR, pages 1-7, 2007.

[8] S. H. Joshi, E. Klassen, A. Srivastava, and I. H. Jermyn. Removing shape-preserving transformations in square-root elastic (SRE) framework for shape analysis of curves. In A. Y. et al., editor, EMMCVPR, LNCS 4679, pages 387-398, 2007.

[9] I. A. Kakadiaris, G. Passalis, G. Toderici, M. N. Murtuza, Y. Lu, N. Karampatziakis, and T. Theoharis. Threedimensional face recognition in the presence of facial expressions : An annotated deformable model approach. IEEE Transactions on Pattern Analysis and Machine Intelligence, 29(4) :640-649, 2007.

[10] E. Klassen and A. Srivastava. Geodesics between 3D closed curves using path-straightening. In Proceedings of ECCV, Lecture Notes in Computer Science, pages I : 95-106, 2006.

[11] E. Klassen, A. Srivastava, W. Mio, and S. Joshi. Analysis of planar shapes using geodesic paths on shape spaces. IEEE Pattern Analysis and Machine Intelligence, 26(3) :372-383, March, 2004.

[12] P. W. Michor, D. Mumford, J. Shah, and L. Younes. A metric on shape space with explicit geodesics. rend.lincei mat.appl., $9: 25,2008$.

[13] P. J. Phillips, P. J. Flynn, T. Scruggs, K. W. Bowyer, J. Chang, K. Hoffman, J. Marques, J. Min, and W. Worek. Overview of the face recognition grand challenge. In IEEE CVPR, pages 947-954, 2005.
[14] C. Samir, A. Srivastava, M. Daoudi, and E. Klassen. An intrinsic framework for analysis of facial surfaces. International Journal of Computer Vision, 80-95(1), 2009.

[15] A. Srivastava, S. H. Joshi, W. Mio, and X. Liu. Statistical shape analysis : Clustering, learning, and testing. IEEE Transactions on Pattern Analysis and Machine Intelligence, 27(4) :590-602, 2005.

[16] P. Yan and K. W. Bowyer. Biometric recognition using 3d ear shape. EEE Transactions on Pattern Analysis and Machine Intelligence, 29(8) :1297-1308, 2007. 